\title{
Regulation of the oncogenic function of distal-less 4 by microRNA-122 in hepatocellular carcinoma
}

\author{
XU-HUA XIE ${ }^{1}$, XIAO-PEI XU ${ }^{2}$, CHANG-YU SUN ${ }^{1}$ and ZU-JIANG YU ${ }^{1}$ \\ ${ }^{1}$ Department of Infectious Diseases, The First Affiliated Hospital of Zhengzhou University, Zhengzhou, Henan 450052; \\ ${ }^{2}$ Department of Endocrinology, Second Hospital of Zhengzhou, Zhengzhou, Henan 450006, P.R. China
}

Received October 31, 2013; Accepted May 9, 2014

DOI: $10.3892 / \mathrm{mmr} .2015 .3554$

\begin{abstract}
Distal-less 4 (DLX4) is a member of the DLX family of homeobox genes. Recent reports have suggested that abnormal expression of DLX4 is present in several types of human tumors, including breast cancer, leukemia and colon cancer. However, the function and the mechanistic regulation of DLX4 in hepatocellular carcinoma (HCC) are elusive. In the present study, a proportion of hepatocellular carcinomas were identified to exhibit upregulated DLX4 expression. This study proposed that the overexpression of DLX4 is associated with the downregulation of miR-122, an underexpressed miRNA in human HCC. Functional studies have demonstrated that the downregulation of DLX4 in hepatocellular carcinoma cell lines is regulated by miR-122 through binding to its 3'UTR. Furthermore, a DLX4 overexpression vector lacking the 3'UTR was shown to abolish miR-122-induced inhibition of proliferation in the HCC cell line Hep3B. These results gave new insight into the mechanism of the miR-122/DLX4 axis in HCC.
\end{abstract}

\section{Introduction}

Distal-less homeobox gene 4 (DLX4) is located on chromosome 17q21-22 and is a member of the DLX family of homeobox genes (1). Although absent in the majority of normal adult tissues, DLX4 has been reported to be widely expressed in leukemia and lung, breast, ovarian and prostate cancers (2-5). The expression of DLX4 mRNA has been shown to be significantly increased in tumors with lymph node metastasis and a high histological grade (6). The expression levels and potential roles of DLX4 in HCC, however, remain to be determined. Hepatocellular carcinoma (HCC), has a high incidence of tumor recurrence and metastasis, and

Correspondence to: Professor Zu-Jiang Yu, Department of Infectious Diseases, The First Affiliated Hospital of Zhengzhou University, 1 Jianshe Road, Zhengzhou, Henan 450052, P.R. China E-mail: yuzujiang779@163.com

Key words: DLX4, miR-122, cell proliferation, hepatocellular carcinoma, cell invasion is considered to be a major worldwide health problem (7). The oncogenic and tumor suppressive functions of numerous genes have been characterized; however, the molecular mechanisms of HCC are complex and remain to be fully elucidated. As a member of the homeobox family, DLX4 may be involved in HCC progression.

MicroRNAs (miRNAs) are a broad class of small, non-coding endogenous single RNA molecules that function in gene expression through directly binding to the 3'-untranslated region ( 3 'UTR) of the target gene mRNA, resulting in mRNA cleavage or translational repression (8). miRNAs are differentially expressed in human cancers and have essential roles in carcinogenesis, including the development of HCC. Microarray analyses have started to identify the numbers of miRNAs that are dysregulated in HCC tissues as compared with normal tissues, including miR-122. It has been reported that microRNA-122 (miR-122), which accounts for $70 \%$ of the total miRNA in the liver, has a central function in the liver (9). However, there are few reports investigating the association between miR-122 and DLX4 in HCC.

In the present study, the expression levels of DLX4 in HCC tissues were investigated and compared with those in the adjacent normal tissues. The association between DLX4 and miR-122 in HCC cancer development was next investigated. The effect of DLX4 knockdown, deletion of the 3'UTR of DLX4 or its inhibition by miR-122 on DLXR levels, cell proliferation and colony formation were investigated. This study may provide a novel insight into the mechanism of miR-122/DLX4 axis in hepatocellular carcinoma.

\section{Materials and methods}

Clinical HCC specimens and RNA isolation. Paired samples of primary HCCs and corresponding adjacent liver tissues from patients were obtained from the First Affiliated Hospital of Zhengzhou University (Henan, China) with the informed consent of the patients. Ethics approval was granted by the Ethics Committee of Zhengzhou University. Total RNA was extracted using the TRIzol ${ }^{\mathrm{TM}}$ Reagent (Invitrogen Life Technologies, Carlsbad, CA), according to the manufacturer's instructions. The average age of the patients, including four males and three females, was 55.0 \pm 5.6 years. Six patients were identified as hepatitis B positive. Tumors with moderate differentiation were identified in five patients 
and tumors with poor differentiation in two patients According to the tumor, nodes and metastasis (TNM) classification of malignant tumors, there was one patient in stage I, three in stage II, and three in stage III or IV.

Cell culture and transfection. Hep3B cells (HB-8064; ATCC Manassas, VA, USA) were cultured in Dulbecco's modified Eagle's medium supplemented with $10 \%$ fetal bovine serum, $100 \mathrm{U} / \mathrm{ml}$ penicillin and $100 \mu \mathrm{g} / \mathrm{ml}$ streptomycin (Gibco-BRL, Grand Island, NY, USA). Hep3B cells were incubated at $37^{\circ} \mathrm{C}$ in a humidified chamber supplemented with $5 \% \mathrm{CO}_{2}$. The miRNA-122 mimic, negative control (NC) and DLX4 siRNA were all purchased from RiboBio (Guangzhou, China). Cells were plated at $30-50 \%$ confluency and transfected with $100 \mathrm{nM}$ miR-122 mimics or negative control using Lipofectamine ${ }^{\text {TM }} 2000$ in Opti-MEM (Invitrogen Life Sciences), according to the manufacturer's instructions.

Cell growth assay. Cells were seeded in 96-well plates at 8,000 cells per well and transfected on the following day. MTT assay was used to determine the relative cell growth at 12,24 , 36 and $48 \mathrm{~h}$ after transfection. A total of $20 \mu \mathrm{l}$ MTT solution was added to $100 \mu \mathrm{l}$ culture media and the cells were incubated for a further $4 \mathrm{~h}$ at $37^{\circ} \mathrm{C}$. The optical density was then measured at $570 \mathrm{~nm}(\mathrm{OD} 570)$.

Colony formation assay. Following transfection, the cells were seeded into 12-well plates at a density of 200 cells/well and the media was changed every three days. After $\sim 10$ days, most of the cell clones contained $>50$ cells. The clones were washed once with phosphate buffered saline and stained with crystal violet for $\sim 5 \mathrm{~min}$. Finally, images were taken of the clones, and the colonies were counted. The colony formation rate $=$ (number of clones) $/$ (number of seeded cells) $\times 100 \%$.

Quantitative polymerase chain reaction $(q P C R)$. The stem-loop qPCR method was used to detect the miR-122 levels in Hep3B cells. The detection of expression levels of DLX4 mRNA was performed as previously described (10). The primer sequence for DLX4 was forward , 5'-CAAAGCTGTCTTCCCAGACC-3'; and reverse, 5'-GTTGTAGGGGACAAGCCAAG-3'. The SYBR $^{\circledR}$ Green Mix Taq ${ }^{\mathrm{TM}}$ kit (Takara, Shiga, Japan) was used to trace the amplified DNA.

Western blot analysis. The Hep3B cells were seeded into six-well plates at a density of $3 \times 10^{5}$ cells/well. The cells were transfected once the density reached $\sim 80 \%$ confluency on the second day. The cells were lysed in radioimmunoprecipitation assay buffer $48 \mathrm{~h}$ after transfection, for $30 \mathrm{~min}$ at $4^{\circ} \mathrm{C}$. The protein concentration was measured by bicinchoninic acid assay and then $20 \mu \mathrm{g}$ protein was separated by SDS-PAGE for further analysis. The primary antibodies used were rabbit polyclonal anti-human DLX4 (1:1,000; Abcam, Cambridge, MA, USA) and rabbit monoclonal anti-human GAPDH (1:1,000; Abcam). The secondary antibody was goat anti-rabbit immunoglobulin $\mathrm{G}$ conjugated with horseradish peroxidase (1:1,000; Abcam). The bound antibodies were detected with the use of Enhanced Chemiluminescence Plus Western Blotting Detection system (GE Healthcare, Buckinghamshire, UK) and the chemiluminiscent signals were detected with the use of high-performance chemiluminescence film (GE Healthcare).

Luciferase reporter assay and vector construction. The 3'-UTR sequence of DLX4 was predicted by TargetScan (www. targetscan.org/) to interact with miR-122. Plasmids containing the DLX4-3'UTR, DLX4-3'UTR with a mutated sequence of the 3'-UTR sequence and DLX4 open reading frame without the 3'UTR were constructed with technical support from Guangzhou Zhiyou Biotech Co. Ltd. (Guangdong, China) and inserted into pGL3 vectors (Promega Corporation, Madison, WI, USA). Following transfection of miR-122 for $24 \mathrm{~h}$, Hep3B cells were transfected with pGL3/DLX4-3'UTR and pGL3/DLX4-3'UTR mutant plasmids. After $48 \mathrm{~h}$ of transfection, the luciferase activity of Hep3B cells was measured using the Dual-Luciferase reporter assay system (Promega Corporation). Construction of the DLX4 siRNA was designed according to a previous study (10).

Statistical analysis. All data are presented as the mean \pm standard deviation, from three independent experiments. Statistical analyses were performed using SPSS 16.0 software (SPSS Inc., Chicago, IL, USA) and statistical significance between treatment and control groups was assessed by analysis of variance or the Student's t-test. A P $<0.05$ was considered to indicate a statistically significant difference.

\section{Results}

DLX4 is downregulated in HCC tissues as compared with the adjacent normal tissues. Previous studies have shown that DLX4 is upregulated in leukemia and lung, breast, ovarian and prostate cancers (2-5). In the present study, seven paired HCC tissues were analyzed by qPCR and western blot to detect the expression status of DLX4. It was identified that both the mRNA and protein expression of DLX4 was increased as compared with the adjacent non-tumor tissues (Fig. 1). These data suggested that DLX4 was upregulated in HCC, implying that DLX4 may act as a tumor oncogene during HCC development. These data demonstrated that the expression of DLX4 in HCC was consistent with expression data obtained from other solid tumors.

DLX4 isoform 1 is targeted by miR-122, which downregulates its expression. TargetScan was used predict miRNAs that may be involved in the regulation of DLX4 expression. As shown in Fig. 2A, 14 candidate miRNAs were identified. Subsequently, a luciferase assay was used to determine which miRNA was the most effective in regulating DLX4. Hep3B cells were transfected with a reporter vector along with the 14 miRNA mimics. The results indicated that miR-122 significantly downregulated the luciferase intensity of pGL3/Luciferase-DLX4-3'-UTR, with miR-138 and miR-216b to a lesser extent (Fig. 2B). Subsequent studies therefore focused on miR-122, a liver specific and the most abundant miRNA in the liver. A second luciferase assay was performed to validate whether the DLX4-3'UTR was the target site through which miR-122 directly regulates DLX4 expression (Fig. 2C). Hep3B cells were transfected with the reporter vector along with miR-122 mimics or NC mimics. The miR-122 mimics significantly 
A

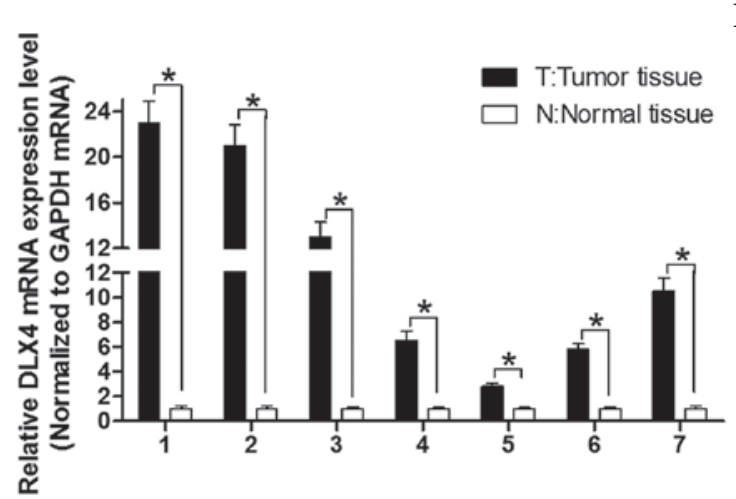

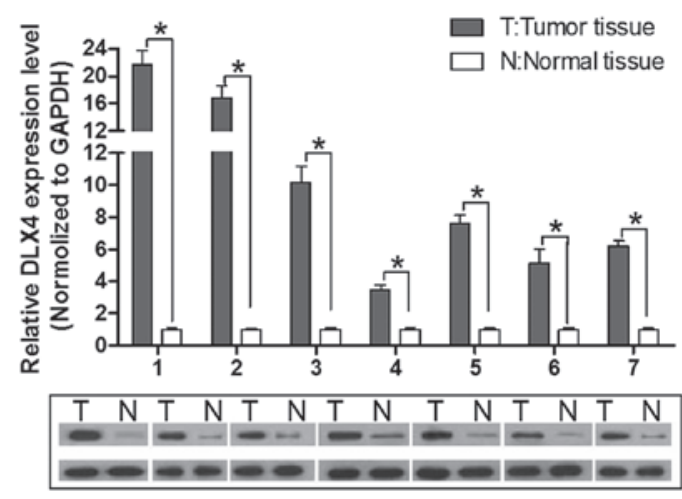

Figure 1. Differential expression of DLX4 in HCC tissues as compared with adjacent normal tissues. (A) Relative expression levels of DLX4 mRNA in the seven liver tissue pairs, including HCC tissues and matched normal tissues, were detected by quantitative polymerase chain reaction. (B) Expression of DLX4 protein in $\mathrm{HCC}$ tissue and normal liver tissues by western blot. All data are expressed as the mean \pm standard deviation of three independent experiments. "P<0.05 as compared with the control group. T, HCC tumor tissue; N, adjacent normal tissue; DLX4, distal-less homeobox 4; HCC, hepatocellular carcinoma.

A

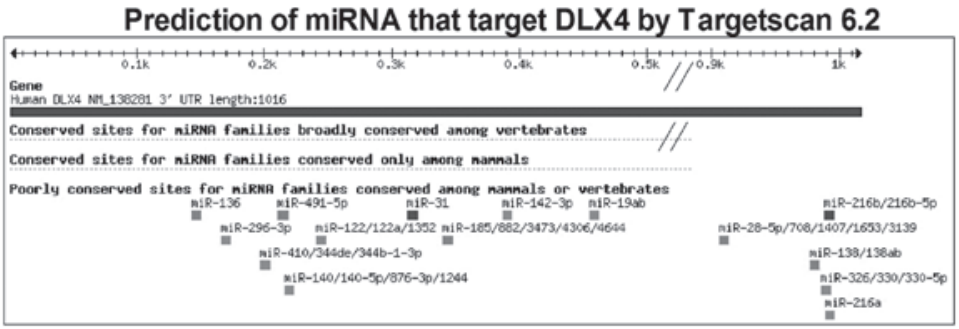

B

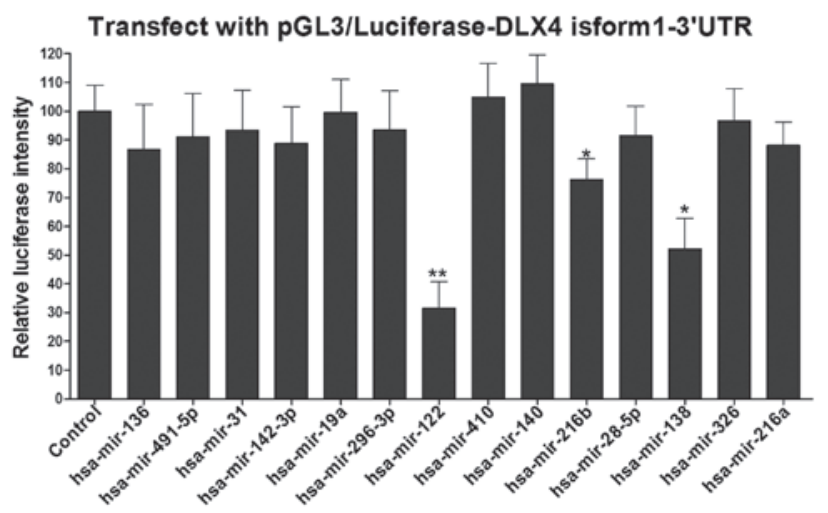

C

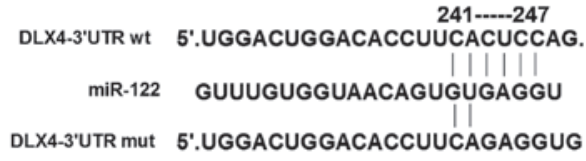

D

NC miR-122Anti-NCAnti-miR122
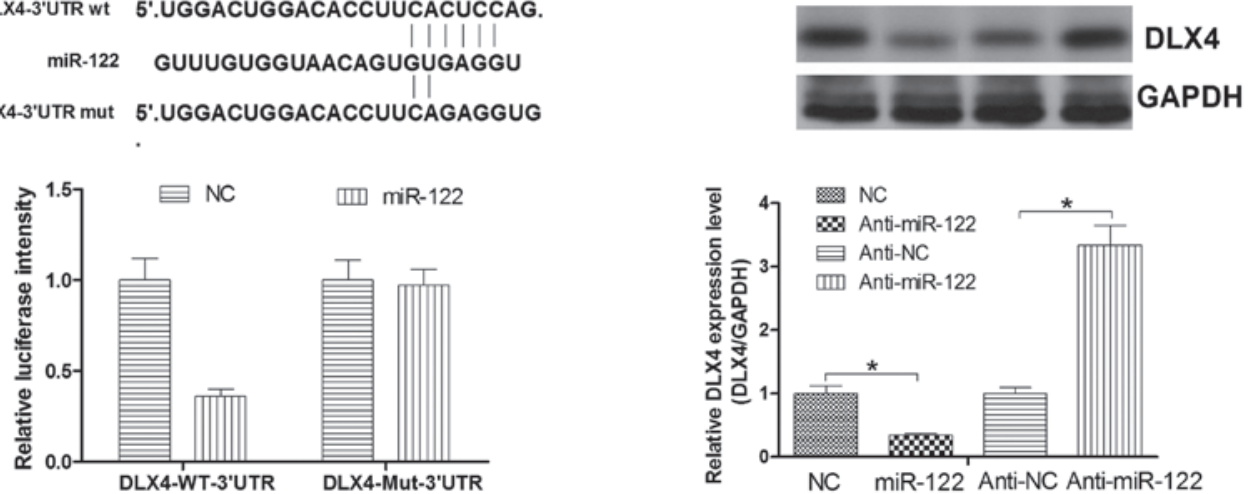

Figure 2. miR-122 directly targets DLX4 and represses DLX4 in Hep3B cells. (A) A schematic representation showing the miRNA target prediction algorithms, generated by Targetscan 6.2, and screen for potential miRNAs that target the DLX4 mRNA 3'UTR. (B) Hep3B cells were co-transfected with luciferase constructs expressing the DLX4-3'UTR (pGL3/luciferase DLX4-3'UTR) and 15 miRNA mimics, including one control mimic. Luciferase activity was determined $48 \mathrm{~h}$ after transfection and normalized to the control. (C) Luciferase constructs were transfected into cells transduced with miR-122 mimics and a negative control. Luciferase activity was determined $48 \mathrm{~h}$ after transfection. The ratio of normalized sensor to control luciferase activity is shown. (D) miR-122 and antisense miR-122 were transfected into Hep3B cells, and then the DLX4 protein levels were measured by western blot analysis. Labwork 4.0 software was used to quantify the intensity of the DLX4 and GAPDH bands. GAPDH was used as a loading control. The level of DLX4 in the NC and Anti-NC group were set as 1.0. All data are expressed as the mean \pm standard deviation of three independent experiments. " $\mathrm{P}<0.05$ as compared with the control group. NC, negative control; WT, wild-type; Mut, mutant; 3'UTR, 3'-untranslated region; DLX4, distal-less homeobox 4; Anti, antisense; miR-122, microRNA-122; hsa, Homo sapiens. 
A

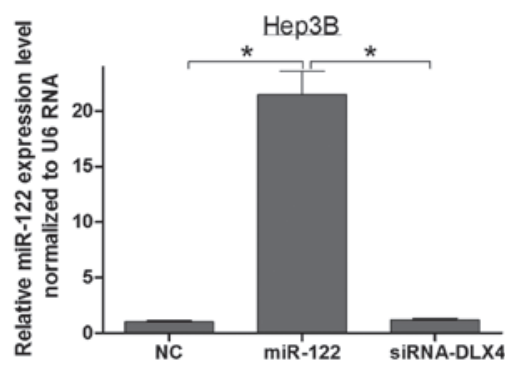

C

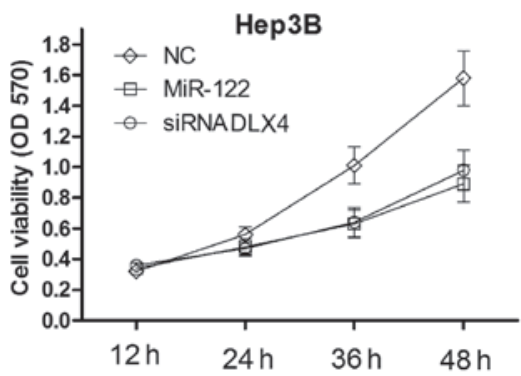

B

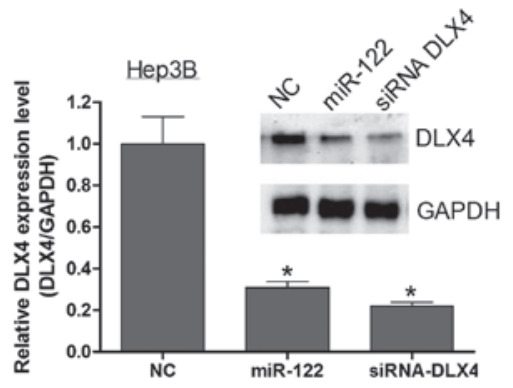

D

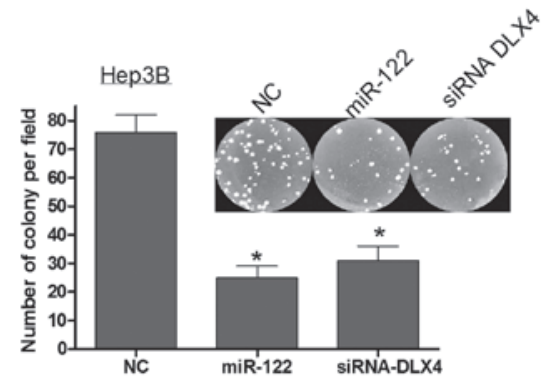

Figure 3. DLX4 modulation accounts for the anti-proliferative effects of miR-122 in Hep3B cells. (A) Validation of miR-122 mimics using quantitative polymerase chain reaction. (B) Western blot analysis of DLX4 protein expression levels in Hep3B cells after $48 \mathrm{~h}$ of miR-122 and DLX4 siRNA transfection. (C) Effects of miR-122 and siRNA DLX4 on the proliferation of Hep3B cells. (D) Effects of miR-122 and siRNA DLX4 on the colony formation ability of Hep3B cells. All data are expressed as the mean \pm standard deviation of three independent experiments. "P $<0.05$ as compared with the control group. NC, negative control; DLX4, distal-less homeobox 4; miR-122, microRNA-122; siRNA, small interfering RNA; OD, optical density; Hep3B, human hepatocellular carcinoma cells.

decreased the luciferase intensity of Hep3B cells transfected with the DLX4-3'UTR reporter vector, whereas it did not affect the luciferase intensity of Hep3B cells transfected with the DLX4-3'UTR mutated vector (Fig. 2C). The data indicated that DLX4 was a direct target of miR-122. The effect of miR-122 on the endogenous expression of DLX4 protein was assayed by western blotting. The DLX4 protein levels increased 2.95-fold in Hep3B cells transfected with miR-122 antisense mimics as compared with the control group (Fig. 2D). The DLX4 protein levels were decreased by 58\% in Hep3B cells transfected with miR-120 mimics as compared with the control group (Fig. 2D). These results indicated that miR-122 targeted and repressed the expression of DLX4, which may explain, in part, the upregulation of DLX4 in HCC.

DLX4 is a critical mediator of the anti-proliferative effects of $m i R-122$. A previous study reported that the overexpression of miR-122 can inhibit HCC cell growth and promote apoptosis (11). Since it was identified that DLX4 was directly targeted by miR-122, it was further explored whether DLX4 may be the critical mediator of the role of miR-122 in cellular proliferation in HCC. Firstly, qPCR and western blot analysis were used to assess the expression of miR-122 and DLX4 in Hep3B cells transfected with miR-122 mimics or siRNA DLX4 (Fig. 3A and B). Silencing the expression of DLX4 using siRNA in Hep3B cells showed that the repression of DLX4 recapitualted the anti-proliferative effects of miR-122 (Fig. 3C and D). These results indicated that DLX4 is a critical mediator of the miR-122-associated anti-proliferative effects in HCC.

Ectopic expression of DLX4 without the 3'UTR counteracts the effects of miR-122 in Hep3B cells. To further confirm that the effects of miR-122 on Hep3B cell proliferation are in part mediated by DLX4, a vector was constructed containing the coding sequence of DLX4 without the 3'UTR to avoid miRNA interference. Firstly, the expression of miR-122 in each Hep3B cell transfection was confirmed (Fig. 4A). Following this, MTT (Fig. 4C), colony formation (Fig. 4D) and western blot assays (Fig. 4B) were used to show that the ectopic expression of DLX4 alleviated the effects caused by miR-122 in Hep3B cells. These results further confirmed that miR-122 suppressed cell viability and colony formation by downregulating DLX4 expression in Hep3B cells.

\section{Discussion}

DLX homeobox genes, originally identified in Drosophila, are transcription factors that regulate the transcription of downstream genes. DLX4 belongs to the DLX group of homeobox genes in humans, and has at least two distinct spliced variants (12). Loss of function studies of DLX4 in breast cancer have strongly implicated a role for this gene in cellular transformation, alterations to the cell cycle and apoptosis, and progression to a metastatic phenotype (12). In the present study, it was demonstrated that DLX4 mRNA and protein were overexpressed in HCC tissues as compared with the adjacent normal tissues, further supporting a role in oncogenesis in solid tumors (Fig. 1). Cavalli et al (13) reported that overexpression of DLX4 protein is caused by gene amplification in tumors. However, the mechanism of DLX4 gene regulation was shown to be complex in HCC.

Previous research has estimated that at least $30 \%$ of protein-coding genes in the human genome are regulated by miRNAs, and that the majority of individual miRNAs target 
A

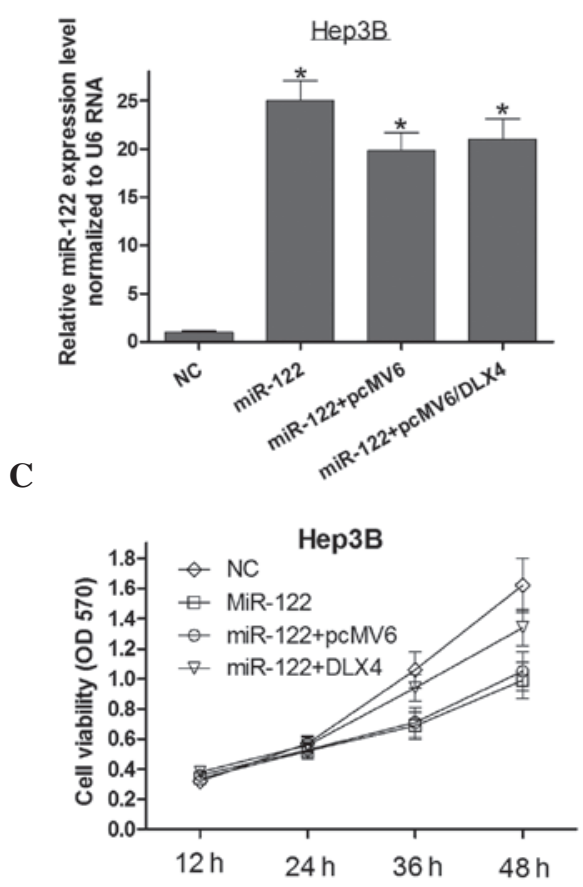

B

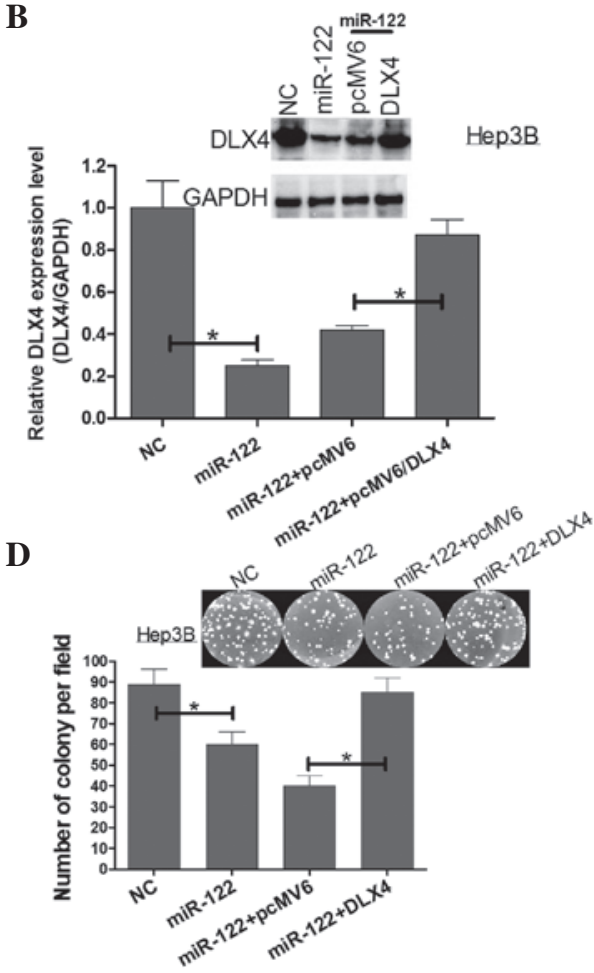

Figure 4. DLX4 alleviates miR-122-induced cellular phenotypes in Hep3B cells. (A) Hep3B cells were co-transfected with the pcMV6/DLX4 vector, which did not contain the 3'-UTR of DLX4, with or without miR-122. Quantitative polymerase chain reaction was used to validate the expression of miR-122 in each group. (B) Western blot analysis was used to validate the expression of DLX4 in each group. (C) Cell viability was detected by MTT assay at 12, 24, 36 and $48 \mathrm{~h}$ after transfection. (D) A colony formation assay was performed following transfection. All data are expressed as the mean \pm standard deviation of three independent experiments. "P<0.05 compared with control group. NC, negative control; DLX4, distal-less homeobox 4; miR-122, microRNA-122; Hep3B, human hepatocellular carcinoma cells; pcMV6, mammalian expression vector; 3'UTR, 3'-untranslated region.

miR-122/DLX4 axis and targets of miR-122 in hepatocellular carcinoma cells

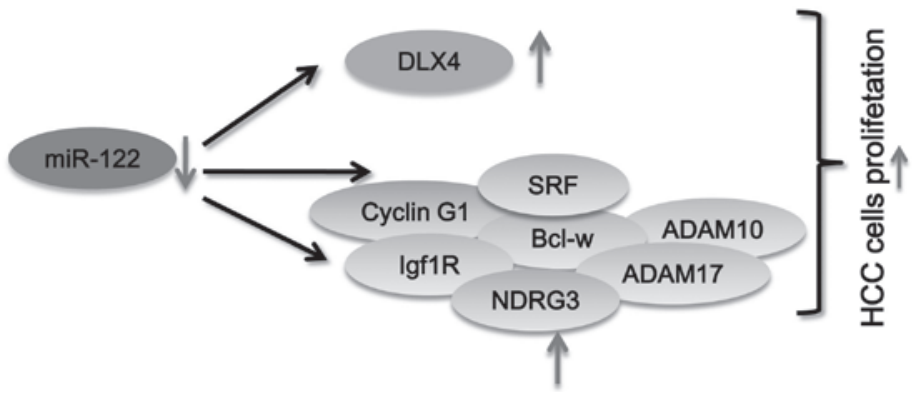

Figure 5. Schematic representation of the hypothetical molecular mechanism of the miRNA-122/DLX4 axis regulating proliferation of HCC cells. HCC, hepatocellular carcinoma; miR-122, microRNA-122; DLX4, distal-less homeobox 4.

multiple protein-coding genes (14). MiRNAs can function as novel types of oncogenes or tumor suppressors and aberrant regulation of specific miRNAs and their targets is associated with tumor cell proliferation, apoptosis, angiogenesis, migration and metastasis (15). In the present study, a liver specific miRNA, miR-122, was identified that can directly target DLX4 and regulate its expression in HCC (Fig. 2). The results presented in this study, for the first time, to the best of our knowledge, suggested that miRNA can participate in the regulation of DLX4 expression via mechanisms other than gene amplification (Fig. 5).

MiR-122 is a liver-specific miRNA, and is the most abundant miRNA in the liver. It acts as a tumor suppressor by binding to target molecules involved in numerous biological processes, including cell proliferation, differentiation, apoptosis and angiogenesis in HCC (16-19). MiR-122 can modulate cyclin G1 expression in HCC-derived cell lines, and an inverse correlation between miR-122 and cyclin G1 expression exists in HCCs, indicating that cyclin G1 is a target of miR-122 (18). MiR-122 also modulates B-cell lymphoma (Bcl)-w expression by directly targeting the binding site within the 3'-UTR. The cellular mRNA and protein levels of Bcl-w were shown to be repressed by elevated levels of miR-122, which subsequently led to a reduction of cell viability and activation of caspase-3. This suggested that Bcl-w is a direct target of miR-122 that functions as an endogenous apoptosis enhancer in HCC cells (16). Other miR-122 target genes include SRF, Igf1R, ADAM10, ADAM17 in HCC (20). In the present study, a new miR-122/DLX4 axis was 
identified that inhibited Hep3B cell colony formation and cell viability. Ectopic expression of DLX4 without the 3'UTR alleviated the inhibition of colony formation and cell viability caused by miR-122 in Hep3B cells. Furthermore, DLX4 knockdown inhibited colony formation and cell proliferation in Hep3B cells. These results were in accordance with the tumor suppressor function of miR-122. These findings strongly support the involvement of the signaling pathway of miR-122 and its target genes in HCC tumorigenesis (Fig. 5).

In conclusion, the present study identified that: (1) DLX4 was upregulated in $\mathrm{HCC}$ tissues as compared with adjacent normal tissues at both the mRNA and protein level. (2) A liver-specific miRNA, miR-122, was identified, which directly targeted and reduced the expression of DLX4 in HCC Hep3B cells. This may contribute to the molecular mechanism of deregulation of DLX4 in HCC. (3) Overexpression of miR-122 or knockdown of the expression of DLX4 caused a marked inhibition of proliferation in Hep3B cells. This phenotype could be rescued by transfection with a DLX4 vector lacking the 3'UTR. These results provided strong evidence that DLX4 was upregulated in HCC and functioned as a tumor suppressor. The data supported that DLX4 is regulated by miR-122, which provides novel insight into the mechanism of the miR-122/DLX4 axis in HCC. Further studies are required to evaluate the function of DLX4 and the miR-122/DLX4 signaling pathway in other tumors.

\section{References}

1. Haga SB, Fu S, Karp JE, et al: BP1, a new homeobox gene, is frequently expressed in acute leukemias. Leukemia 14: $1867-1875,2000$.

2. Hara F, Samuel S, Liu J, et al: A homeobox gene related to Drosophila distal-less promotes ovarian tumorigenicity by inducing expression of vascular endothelial growth factor and fibroblast growth factor-2. Am J Pathol 170: 1594-1606, 2007.

3. Man YG, Fu SW, Schwartz A, et al: Expression of BP1, a novel homeobox gene, correlates with breast cancer progression and invasion. Breast Cancer Res Treat 90: 241-247, 2005.

4. Man YG, Schwartz A, Levine PH, Teal C and Berg PE: BP1, a putative signature marker for inflammatory breast cancer and tumor aggressiveness. Cancer Biomark 5: 9-17, 2009.
5. Schwartz AM, Man YG, Rezaei MK, Simmens SJ and Berg PE: $\mathrm{BP} 1$, a homeoprotein, is significantly expressed in prostate adenocarcinoma and is concordant with prostatic intraepithelial neoplasia. Mod Pathol 22: 1-6, 2009.

6. Yu M, Wan Y and Zou Q: Prognostic significance of BP1 mRNA expression level in patients with non-small cell lung cancer. Clin Biochem 41: 824-830, 2008.

7. Parkin DM: The global health burden of infection-associated cancers in the year 2002. Int J Cancer 118: 3030-3044, 2006.

8. Hutvágner $G$ and Zamore PD: A microRNA in a multiple-turnover RNAi enzyme complex. Science 297: 2056-2060, 2002

9. Tsai WC, Hsu SD, Hsu CS, et al: MicroRNA-122 plays a critical role in liver homeostasis and hepatocarcinogenesis. J Clin Invest 122: 2884-2897, 2012.

10. Kluk BJ, Fu Y, Formolo TA, et al: BP1, an isoform of DLX4 homeoprotein, negatively regulates BRCA1 in sporadic breast cancer. Int J Biol Sci 6: 513-524, 2010.

11. Xu J, Zhu X, Wu L, et al: MicroRNA-122 suppresses cell proliferation and induces cell apoptosis in hepatocellular carcinoma by directly targeting Wnt/ $\beta$-catenin pathway. Liver Int 32: 752-760, 2012.

12. Fu S, Stevenson H, Strovel JW, et al: Distinct functions of two isoforms of a homeobox gene, BP1 and DLX7, in the regulation of the beta-globin gene. Gene 278: 131-139, 2001.

13. Cavalli LR, Man YG, Schwartz AM, et al: Amplification of the BP1 homeobox gene in breast cancer. Cancer Genet Cytogenet 187: 19-24, 2008.

14. Lewis BP, Burge CB and Bartel DP: Conserved seed pairing, often flanked by adenosines, indicates that thousands of human genes are microRNA targets. Cell 120: 15-20, 2005.

15. Esquela-Kerscher A and Slack FJ: Oncomirs - microRNAs with a role in cancer. Nat Rev Cancer 6: 259-269, 2006.

16. Lin CJ, Gong HY, Tseng HC, Wang WL and Wu JL: miR-122 targets an anti-apoptotic gene, Bcl-w, in human hepatocellular carcinoma cell lines. Biochem Biophys Res Commun 375: 315-320, 2008

17. Ma L, Liu J, Shen J, et al: Expression of miR-122 mediated by adenoviral vector induces apoptosis and cell cycle arrest of cancer cells. Cancer Biol Ther 9: 554-561, 2010.

18. Gramantieri L, Ferracin M, Fornari F, et al: Cyclin G1 is a target of miR-122a, a microRNA frequently down-regulated in human hepatocellular carcinoma. Cancer Res 67: 6092-6099, 2007.

19. Wu X, Wu S, Tong L, et al: miR-122 affects the viability and apoptosis of hepatocellular carcinoma cells. Scand J Gastroenterol 44: 1332-1339, 2009.

20. Saito Y, Suzuki H, Matsuura M, et al: MicroRNAs in Hepatobiliary and Pancreatic Cancers. Front Genet 2: 66, 2011. 